\title{
Corrigendum: Development and Validation of a Prognostic Nomogram to Predict Cancer-Specific Survival in Adult Patients With Pineoblastoma
}

\author{
Yajun Jing ${ }^{1}$, Wenshuai Deng ${ }^{1}$, Huawei Zhang ${ }^{1,2}$, Yunxia Jiang ${ }^{3}$, Zuoxiang Dong ${ }^{1}$, \\ Fan Fan ${ }^{2 *}$ and Peng Sun ${ }^{1 *}$ \\ ${ }^{1}$ Department of Neurosurgery, Affiliated Hospital of Qingdao University, Qingdao, China, ${ }^{2}$ Department of Pharmacology and \\ Toxicology, University of Mississippi Medical Center, Jackson, MS, United States, ${ }^{3}$ Department of Nursing, Medical College \\ of Qingdao University, Qingdao, China
}

Keywords: pineoblastoma, nomogram, prognosis, risk factor, C-index

\section{A Corrigendum on}

\section{OPEN ACCESS}

Edited and reviewed by: Theodore Nicolaides, New York University, United States

*Correspondence:

Fan Fan

ffan@umc.edu

Peng Sun

sunpeng@qdu.edu.cn

Specialty section:

This article was submitted to Neuro-Oncology and Neurosurgical

Oncology,

a section of the journal

Frontiers in Oncology

Received: 12 August 2020

Accepted: 14 August 2020

Published: 02 October 2020

Citation:

Jing $Y$, Deng $W$, Zhang $H$, Jiang $Y$, Dong Z, Fan F and Sun P (2020)

Corrigendum: Development and Validation of a Prognostic Nomogram to Predict Cancer-Specific Survival in Adult Patients With Pineoblastoma.

Front. Oncol. 10:594049.

doi: 10.3389/fonc. 2020.594049
Development and Validation of a Prognostic Nomogram to Predict Cancer-Specific Survival in Adult Patients With Pineoblastoma

by Jing, Y., Deng, W., Zhang, H., Jiang, Y., Dong, Z., Fan, F., et al. (2020). Front. Oncol. 10:1021. doi: $10.3389 /$ fonc.2020.01021

In the original article, there were two mistakes in the legends for the Kaplan-Meier curves and the nomogram as published. The Kaplan-Meier curves in the section Independent Prognostic Factors in the Primary Cohort were supposed to be Figure 4, but were incorrectly included as Figure 5. In addition, the nomogram in the section Prognostic Nomogram of Overall Survival was supposed to be Figure 5, but it was incorrectly included as Figure 4. The correct legends appear below.

Figure $4 \mid$ Kaplan-Meier curves for patients with PB according to different independent prognostic factors. The Kaplan-Meier curves for patients with primary PB according to age (A), year of diagnosis (B), tumor size (C), RT after GTR \& CT or RT after subtotal resection \& CT (D), GTR and subtotal resection (E), RT after GTR or subtotal resection (F), RT, RT after surgery \& RT after surgery \& CT $(\mathbf{G})$, extent of tumor extension $(\mathbf{H})$.

Figure 5 | Nomogram predicting 36-, 60-, and 120-month cancer-specific survival for patients with PB. Prognostic factors including age, race, tumor extension, tumor size, and therapy, and the scores assigned on the points scale could match each level of every variable on the nomogram. Thus, a total score was obtained by adding the score from various variables or their levels. Finally, the 36-, 60-, and 120-month cancer-specific survival for each individual patient could be estimated on the basis of the total score. ${ }^{*} p<0.05,{ }^{* *} p<0.01,{ }^{* * *} p<0.001$.

In the original article, there was an error. The nomogram referenced in the section Comparison of Predictive Accuracy Between the Nomogram and a Single Independent Factor was supposed to be Figure 5, but it was incorrectly written as Figure 4.

A correction has been made to the Results section, sub-section Comparison of Predictive Accuracy Between the Nomogram and a Single Independent Factor, paragraph 1:

The weights of extension and year of diagnosis for survival, shown in Figure 5, were higher than those of other factors. We compared the predictive power for the prognosis of patients 
with $\mathrm{PB}$ between the nomogram, scope of tumor extension, and year of diagnosis. C-indices for the prediction of prognosis by the scope of tumor extension and year of diagnosis were 0.72 and 0.63 , respectively. These values were significantly lower than the C-index obtained through the nomogram $(0.802 ; P<0.01)$.

The authors apologize for these errors and state that this does not change the scientific conclusions of the article in any way. The original article has been updated.

Copyright (c) 2020 Jing, Deng, Zhang, Jiang, Dong, Fan and Sun. This is an openaccess article distributed under the terms of the Creative Commons Attribution License (CC BY). The use, distribution or reproduction in other forums is permitted, provided the original author(s) and the copyright owner(s) are credited and that the original publication in this journal is cited, in accordance with accepted academic practice. No use, distribution or reproduction is permitted which does not comply with these terms. 